periment, because when qualitatively different instances are averaged, artifacts are produced. Elimination of bias from the experimental situation is therefore an irreplaceable requirement for hypothesis-testing ${ }^{2}$. The relative indifference to experimental control, which is intrinsic to the mega-trial methodology (such as the assertion that 'relative risks travel well') is profoundly unscientific.

Most mega-trials provide a highly precise measure of the results of allocating a protocol to an unrepresentative and heterogeneous group of subjects. This measure should not be interpreted as an estimate of therapeutic effect, nor can it be applied to individuals (the average outcome of a heterogeneous group tells us nothing about the individual outcomes in the subjects which compose that group), nor can the measure be assumed to apply to other populations. The typical mega-trial therefore stands in a subordinate role to medical science: science supplies an essential framework without which the trial is uninterpretable.

Overvaluation and misinterpretation of RCTs is currently rampant, particularly in the UK, under the banner of 'Evidence Based Medicine'". Merely because an experiment is big, slow and expensive does not mean it is rigorous. Valid answers are not a consequence of specific techniques. If misleading measurement is the enemy then many clinical trials, far from being the best kind of science, are actually the worst kind of epidemiology.

\section{BRUCE G. CharltoN}

Department of Epidemiology and Public Health

University of Newcastle upon Tyne

Newcastle upon Tyne, NE2 4HH, UK

1. Holmberg, L. \& Baum, M. Can results from clinical trials be generalized? Nature Med. 1, 734-736 (1995).

2. Charlton, B.G. Mega-trials: Methodological issues and clinical implications. J. Royal College Physicians of London 29, 96-100 (1995).

3. Schwartz, D. \& Lellouch, J. Explanatory and pragmatic attitudes in therapeutic trials. J. chron. Dis. 20, 637-648 (1967)

4. Charlton, B.G. The scope and nature of epidemiology. J. clin. Epidemiol. (in the press).

5. Chessells, J.M., Bailey, C., Richards, S.M. \& MRC Working Party on Childhood Leukaemia. Intensification of treatment and survival in all children with lymphoblastic leukaemia: Results of UK Medical Research Council trial UKALL X. Lancet 345, 143-148 (1995)

6. Bernard, C. An Introduction to the Study of Experimental Medicine (Dover, New York, 1865; (1957 edition).

7. Rosenberg, W. \& Donald, A. Evidence based medicine: An approach to clinical problem solving. Br. Med. J. 310, 1122-126 (1995).

\title{
Animal rights and wrongs
}

To the editor - Peggy Carlson condemns the American Academy of Neurology for their distribution of a brochure describing the value of animal research intended to redress the misinformation promulgated by the well-funded animal rights groups (Nature Med. 1, 849; 1995). Carlson claims that the scientific value of animal research is contested by an increasing number of scientists and physicians. Her evidence for this consists of quoting, for example, Kaufman's view that animal models of neurological disorders are of dubious value. Kaufman, incidentally, is not a neurologist, but an ophthalmologist, and is well known as an antivivisectionist. No consideration is given to the fact that animal experiments produced the current drug treatments for epilepsy and Parkinson's disease, and similar experiments are gradually improving our knowledge of the aetiology of Alzheimer's disease.

Carlson's believes that "an examination of the history of medicine shows that animal experiments have not been a significant source of knowledge useful to improving human health", but she must have a very unconventional view of what is significant. Animal experimentation has provided immunotherapy against, for example, smallpox, anthrax (in farm animals), rabies, poliomyelitis, tetanus, pertussis and meningococcal and $\mathrm{Hib}$ meningitis. The life-saving technique of dialysis was developed in rabbits and dogs in 1913 and became routine in patients after the isolation of the necessary anticoagulant heparin from cows and pigs. The use of immunosuppressants to prevent rejection stemmed from research performed on rabbits and dogs and subsequently enabled renal transplantation to become a routine surgical procedure. Similarly, it is hardly conceivable that anyone could believe that the enormous surgical problems involved in cardiopulmonary bypass, which enables the performance of open heart surgery, could have been overcome without the extensive experiments on dogs that occurred between 1933 and 1953, when the first successful use of this technique took place.

Carlson is right to highlight concern for care of laboratory animals. The scientific community should ensure that animals are treated humanely. Comprehensive regulations governing treatment of laboratory animals exist in the UK. These should be rigorously enforced and trans- gressors punished accordingly. However, the total income of the major animal rights groups in the United States is more than $\$ 80$ million per year (anon. Physiologist 38, 149 (1995)). If the Physicians Committee for Responsible Medicine believes there are more relevant techniques to establish treatments for neurological and other conditions, they should persuade their wealthy donors to provide the funding to develop them. If this were achieved, it would provide a more cogent argument for their cause than the belittlement of the undoubted progress in clinical treatment built upon animal experimentation.

\section{J.H. BotTING}

$R D S$, Understanding Animal Research in Medicine 58 Great Marlborough Street

London W1V 1DD, UK

\section{The value of a family physician}

To the editor - I am writing in regard to the news item by Colleen Sauber, in the September issue of Nature Medicine (1, 858; 1995). As a family physician in a rural area, who delivers babies, takes care of families and generally cares for the immediate medical needs of the community, I resent the implication that family physicians are a lower form of life than specialists, as suggested by the comments regarding the 'dumbing-down' of the medical profession, attributed to Jane Orient. It is, I believe, much easier to find security in some small niche specialty area, instead of rising to the challenge of taking care of all the patients that present themselves to a family practitioner. Studies have proven that we practice more cost-effectively, saving the community money and treating the whole person, not just an organ system.

Bodo W. TREU

Buena Vista Clinic, 620 Northwestern

Storm Lake, Iowa 50588, USA

Nature Medicine responds - We agree that the use of "dumbing-down" is to be regretted in this context. It was used as a quote clearly attributed to another group, and we do not endorse the sentiment it encourages. 